\title{
Fluctuations around equilibrium laws in ergodic continuous-time random walks
}

\author{
Johannes H. P. Schulz and Eli Barkai \\ Department of Physics, Institute of Nanotechnology and Advanced Materials, Bar-Ilan University, Ramat Gan 52900, Israel
}

(Dated: September 26, 2018)

\begin{abstract}
We study occupation time statistics in ergodic continuous-time random walks. Under thermal detailed balance conditions, the average occupation time is given by the Boltzmann-Gibbs canonical law. But close to the non-ergodic phase, the finite-time fluctuations around this mean are large and nontrivial. They exhibit dual time scaling and distribution laws: the infinite density of large fluctuations complements the Lévy-stable density of bulk fluctuations. Neither of the two should be interpreted as a stand-alone limiting law, as each has its own deficiency: the infinite density has an infinite norm (despite particle conservation), while the stable distribution has an infinite variance (although occupation times are bounded). These unphysical divergences are remedied by consistent use and interpretation of both formulas. Interestingly, while the system's canonical equilibrium laws naturally determine the mean occupation time of the ergodic motion, they also control the infinite and Lévy-stable densities of fluctuations. The duality of stable and infinite densities is in fact ubiquitous for these dynamics, as it concerns the time averages of general physical observables.

PACS numbers: 05.40.-a, 64.60.F-, 05.10.Gg
\end{abstract}

\section{INTRODUCTION}

Stochastic theories of motion are a well-established approach to model physical trajectories of single particles embedded in a thermal environment. The ergodic motions in particular are an essential prerequisite of statistical mechanics, as they exhibit the following convergence. Let, for a given trajectory, the occupation time $T_{\mathcal{D}}(t)$ designate the total amount of time the particle has spent within a domain $\mathcal{D}$ up to time $t$. Then

$$
\lim _{t \rightarrow \infty} \frac{T_{\mathcal{D}}(t)}{t}=P_{\mathcal{D}}
$$

$P_{\mathcal{D}}$ is the steady-state probability for the occupation of $\mathcal{D}$. For example, for the overdamped motion of a Brownian particle immersed in water at temperature $\mathcal{T}$ and subject to a static, confining potential $V_{x}$, the Boltzmann-Gibbs canonical law yields $P_{\mathcal{D}} \propto$ $\sum_{x \in \mathcal{D}} \exp \left[-V_{x} /\left(k_{\mathrm{B}} \mathcal{T}\right)\right] ; k_{\mathrm{B}}$ is Boltzmann's constant.

In ensemble theories, the focus rests on ensemble laws such as $P_{\mathcal{D}}$. But in practice, the measurement time $t$ is finite, so it is natural to investigate also the fluctuations

$$
\Delta T_{\mathcal{D}}(t)=T_{\mathcal{D}}(t)-t P_{\mathcal{D}}
$$

Knowing their precise distribution is relevant, e.g., when tracking single particles by confocal microscopy or when studying reaction-diffusion dynamics [1-3]. A submanifold of the complete phase space is considered in the problem of phase persistence [4, 5]. The time a laser-cooled atom resides in the "dark" low-momentum state [6, 7] determines the cooling efficiency. In a broader context, fluctuation theorems [8, 9] describe deviations from equilibrium and refine our understanding of thermodynamic laws. As a rule of thumb, occupation time fluctuations are particularly large in the presence of annealed [10 12 or quenched [13, 14] disorder. The essential questions in all cases are: how large are the fluctuations, how do they evolve with time, and how are they determined by the precise microscopic dynamics on the one hand and the universal equilibrium laws $P_{\mathcal{D}}$ on the other?

We study here the site occupation times $T_{x}(t)$ of a confined continuous-time random walk (CTRW) on a lattice, $x \in \mathbb{Z}$. CTRWs and related models are a standard theoretical approach to describe dynamics where trapping mechanisms induce a variety of remarkable motion patterns; examples are the charge carrier transport in amorphous semiconductors [15], model glasses [10], subrecoil laser cooling [6, 7], atomic transport in optical lattices [16] and diffusion in biological cells [17 20], to name a few $[21,26]$. We focus on a regime where the ergodic convergence (11) holds and the classical Boltzmann-Gibbs equilibrium and ergodicity ideas apply. Remarkably, the equilibrium probability $P_{x}$ even controls the distribution of finite-time fluctuations (2).

But close to the non-ergodic regime, these fluctuations can be unexpectedly large, and their distribution exhibits a non-trivial, dual behavior. Firstly, the Lévy-Gauss central limit theorem suggests a standard scaling approach, which yields the non-Gaussian Lévy-stable laws. Secondly, it turns out that we have to explicitly consider the large fluctuations, which deviate from the central limit theorem. To study this aspect, we propose a nonstandard scaling procedure. It leads to a concept which may appear surprising in a probabilistic model: the infinite density. This density is not normalizable, despite being a limit of a properly normalized probability law. While such objects play a key role in the mathematical field of infinite ergodic theory [27 29], their usage is less common in physical models, such as subdiffusion on intermittent maps 30 33], diffusion in logarithmic potentials $34-36$ and recently strong anomalous diffusion 37]. Our goal is to showcase the scope and applicability of infinite densities and familiarize the reader with its peculiar properties. We integrate it in the statistical mechanics framework by relating all our findings to the system's equilib- 
rium statistics $P_{x}$. Finally, we extend our discussion to include the statistics of time-averaged observables. This exposes the ubiquity of the interplay between dual scaling, stable laws and infinite densities in ergodic CTRWs.

We start by introducing the CTRW in Sec. II and briefly review the concept of equilibrium in the CTRW context. The basic nature of the time scaling duality is explained in Sec. III] After a general introduction to the calculation of occupation time statistics and their longtime asymptotic approximation in Sec. IV, we discuss in detail the emergent stable law of bulk fluctuations in Sec. $[\nabla$ and the infinite density of large fluctuations in Sec. VI. Section VII tackles the question of how to apply these dual limiting laws when it comes to ensemble averaging. In Sec. VIII, we consider fluctuations of a time average of an arbitrary observable. To conclude, we summarize our findings and discuss briefly further directions of research, in particular models and applications beyond CTRW, in Sec. IX].

\section{THE CTRW MODEL AND THERMAL EQUILIBRIUM}

We consider a CTRW evolving along a one-dimensional lattice. To each accessible lattice point $x \in \mathbb{Z}$ we assign a probability $0<q_{x}<1$ to make a jump to the right neighboring site; $1-q_{x}$ for jumps to the left. The jumps are instantaneous, but in between jumps, the particle resides on the lattice site for a random trapping or waiting time. All jumps and waiting times are assumed to be statistically independent. The key quantity here is the common probability density function (PDF) $\psi(\tau)$ of waiting times $\tau$, which reflects the disorder and heterogeneity of the medium. According to CTRW theory [25], the long-time qualities of the motion are related to the width of $\psi(\tau)$; more precisely, to the finiteness of its moments $\left\langle\tau^{n}\right\rangle=\int_{0}^{\infty} \tau^{n} \psi(\tau) d \tau$. Motivated by the complex systems collected in the introduction, we consider here distributions with power law tails, i.e. for large $\tau$

$$
\psi(\tau) \sim \frac{A}{|\Gamma(-\alpha)| \tau^{1+\alpha}},
$$

with the coefficient $A>0$, bearing the unit $\sec ^{\alpha}$. Some experimental setups permit the fine-tuning of the tail exponent $\alpha$ via physical parameters such as temperature [19] or particle size [20]. If the tail exponent is from the range $0<\alpha<1$, the mean waiting time $\langle\tau\rangle$ diverges. This regime has been investigated thoroughly in the past, as it gives rise to a wealth of anomalous phenomena [10, 38 40]. We focus on $1<\alpha<2$, where waiting times do possess a characteristic relaxation scale, namely the mean $\langle\tau\rangle<\infty$. Consequently, as shown below, a confined CTRW is ergodic in the sense of Eq. (1). In this context, $P_{x}$ is the probability to find one random walker, picked from a large ensemble of non-interacting random walkers, on position $x$ after a large relaxation

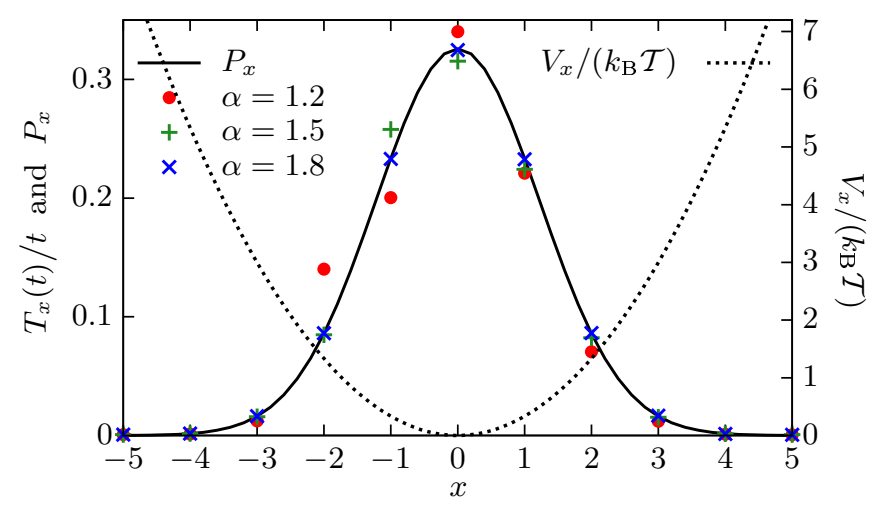

Figure 1. Occupation time fractions $T_{x}(t) / t$ of a bounded, thermal CTRW in comparison with ensemble occupation probabilities $P_{x}$. The waiting time distribution is as in Eq. (3), with $A=0.5$ and $\langle\tau\rangle=1$ (arbitrary units). The statistics of three different paths are shown (symbols), each for one value of $1<\alpha<2$ (see key). The $P_{x}$ are Boltzmann's equilibrium law, Eq. (5), for the confining harmonic potential $V_{x} /\left(k_{\mathrm{B}} \mathcal{T}\right)=x^{2} / 3$. The occupation time fractions $T_{x}(t) / t$ roughly fall onto ensemble laws $P_{x}$. But especially for the smaller $\alpha$, the deviations are huge, considering the long simulation time $t=10^{6}$.

time $t /\langle\tau\rangle \rightarrow \infty$. But while the statement (1) is mathematically concise and correct, it may conceal physical reality. Figure 1 compares occupation time fractions $T_{x}(t) / t$ with ensemble probabilities $P_{x}$ for a CTRW particle which is bound by a harmonic potential $V_{x} \propto x^{2}$ and coupled to a heat bath at temperature $\mathcal{T}$. In this case, the Boltzmann-Gibbs canonical ensemble laws imply a Gaussian form of $P_{x}$ - we discuss thermal systems in more detail below. In Fig. 1, we observe a basic agreement with Eq. (11) on average, but deviations are significant at the huge, yet finite process time $t /\langle\tau\rangle=10^{6}$. The cause for the scatter are the waiting time statistics (3), which, with $1<\alpha<2$, are so broad that $\left\langle\tau^{2}\right\rangle=\infty$. We note that Kac's theorem [41] in chaos theory [42] suggests that Hamiltonian systems should be studied under the premise $\langle\tau\rangle<\infty$, while $\left\langle\tau^{2}\right\rangle$ might indeed be infinite. Hence, we conduct below a detailed analysis of the occupation time fluctuations (2).

Under confinement, the probability to find the random walker at a site $x$ converges with time $t$ to the $t$ independent value $P_{x}$. In this stationary state, the detailed balance condition holds,

$$
q_{x} P_{x}=\left(1-q_{x+1}\right) P_{x+1}, \quad x \in \mathbb{Z} .
$$

The existence of a stationary limiting state and detailed balance conditions are a premise for all CTRW dynamics studied in this work. We may, in particular, choose to consider thermal CTRWs. Then, the hopping probabilities satisfy [12, 23] $q_{x} /\left(1-q_{x+1}\right)=$ $\exp \left[-\left(V_{x+1}-V_{x}\right) /\left(k_{\mathrm{B}} \mathcal{T}\right)\right], x \in \mathbb{Z}$. The aim of a thermal model is to describe systems where the particle is confined by an external potential $V_{x}$ and in contact with 

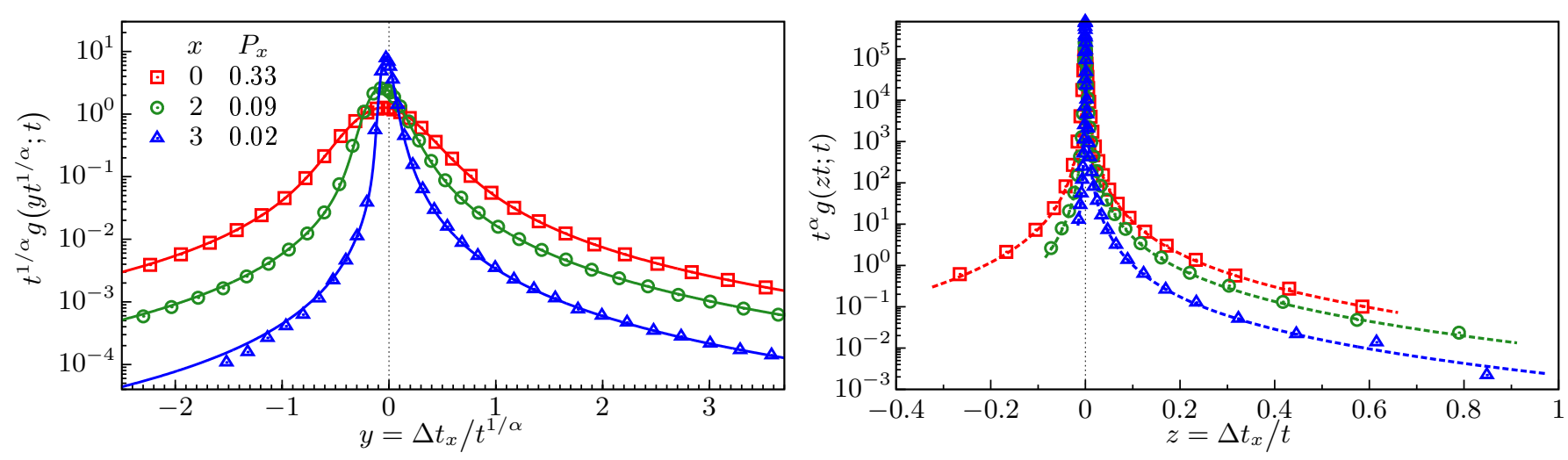

Figure 2. Scaled PDF of occupation time fluctuations $\Delta T_{x}(t)$ for confined CTRW. Symbols in both panels are the simulation results from $10^{7}$ trajectories with $\alpha=1.5$, evaluated on three lattice sites $x \in\{0,2,3\}$ (see key). All other parameters are as in Fig. 1 Both panels depict the same data. But depending on the time scaling we use, different forms of asymptotic behavior become apparent. Left: Scaling with $t^{1 / \alpha}$ as in Eq. (6) yields the asymmetric, stable bulk statistics $g_{\mathrm{B}}(\cdot)$ of Eq. (17) (continuous lines). The agreement between the asymptotic theory and the data is excellent. Right: The nonstandard time scaling in Eq. (7) produces the infinite density $g_{\mathrm{L}}(\cdot)$ for the large fluctuations as in Eq. (25) (dashed lines). These asymptotics are not normalizable due to the blow-up at the origin.

a heat bath at constant temperature $\mathcal{T}$. Detailed balance then implies Boltzmann's canonical distribution

$$
P_{x}=\frac{1}{Z} \exp \left(-\frac{V_{x}}{k_{\mathrm{B}} \mathcal{T}}\right), \quad Z=\sum_{x} \exp \left(-\frac{V_{x}}{k_{\mathrm{B}} \mathcal{T}}\right)
$$

Equations (4)-(5) define a mapping between the sets $\left\{q_{x}\right\},\left\{P_{x}\right\}$ and $\left\{V_{x}\right\}$, thus relating microscopic CTRW dynamics to the Boltzmann-Gibbs equilibrium concept. Recipes for the implementation in numerical simulations and a careful account on the effects of the lattice discretization can be found in Ref. [12], Sec. VIa.

\section{THE DUAL TIME SCALING OF OCCUPATION TIMES}

Let $T_{x}(t)$ be the occupation time at fixed $x$ up to time $t$ since the start of the random walk at time 0 . We denote with $g\left(\Delta t_{x} ; t\right)$ the PDF of fluctuations $\Delta T_{x}(t)$ as in Eq. (2). Its exact form is complicated and depends in particular on initial conditions, the full waiting time PDF $\psi(\tau)$ and the hopping probabilities $q_{x}$. But close to equilibrium, we expect to find a scaling limit form in terms of long-time properties of the process, such as the tail (3) and the equilibrium statistics $P_{x}$. Surprisingly, the occupation times of CTRW feature not one, but two such asymptotic scaling forms. We derive below a bulk ("B") scaling limit

$$
g\left(\Delta t_{x} ; t\right) \sim \frac{1}{t^{1 / \alpha}} g_{\mathrm{B}}\left(\frac{\Delta t_{x}}{t^{1 / \alpha}}\right),
$$

while the large ("L") fluctuations scaling limit is

$$
g\left(\Delta t_{x} ; t\right) \sim \frac{1}{t^{\alpha}} g_{\mathrm{L}}\left(\frac{\Delta t_{x}}{t}\right) .
$$

Figure 2 demonstrates that these scaling laws do indeed coexist. The scaling functions $g_{\mathrm{B}}(\cdot)$ and $g_{\mathrm{L}}(\cdot)$ are derived and discussed in Secs. $\mathrm{V}$ and VI, respectively. But before going deeply into the analytical study, we want to develop an understanding for the character of this duality.

The total occupation time is a sum of independent waiting times with the broad statistics (3). For the bulk, that is, the large majority of process realizations, the number of waiting times entering this sum is large, as its average is $t /\langle\tau\rangle$. By virtue of the generalized central limit theorem [43], one can thus indeed anticipate a scaling limit of the form of Eq. (6). We note that the bulk relation (6) also has a common shorthand notation: $\Delta T_{x}(t) \sim t^{1 / \alpha}$. Relations of this form appear in abundance in the physical and other sciences (with various $g_{\mathrm{B}}(\cdot)$ and $\alpha$ ). It implies a strong statement on the asymptotic statistics: let a threshold $c(t)>0$ increase faster than $t^{1 / \alpha}$; then the probability to observe $\left|\Delta T_{x}(t)\right|>c(t)$ goes to zero with $t$. In this sense, the scope of the approximation $g_{\mathrm{B}}(\cdot)$ widens, and deviations from bulk behavior become rare. Hence, in many other physical contexts, a scaling relation of this type fully describes all asymptotic aspects of the respective process.

But for the CTRW problem at hand, the bulk analysis misses essential features of the distribution. The broad waiting time PDF (3) implies that the rare, large occupation times are relevant events. Since we can in principle have $0 \leq T_{x}(t) \leq t$, the largest occupation times can be of the order of the measurement time $t$ itself. This is why we divide $\Delta t_{x}$ by $t$ in the large fluctuations scaling limit, Eq. (7). But notice that the latter has a nonstandard, counter-intuitive form. The involved scaling function $g_{\mathrm{L}}(\cdot)$ is not properly normalized: integrating Eq. (7) over all $\Delta t_{x}$ gives 1 on the left-hand side, but on the right-hand side we get the seemingly time-dependent value $\int t^{-\alpha} g_{\mathrm{L}}\left(\Delta t_{x} / t\right) d\left(\Delta t_{x}\right) \equiv t^{1-\alpha} \int g_{\mathrm{L}}(z) d z$. In fact, 

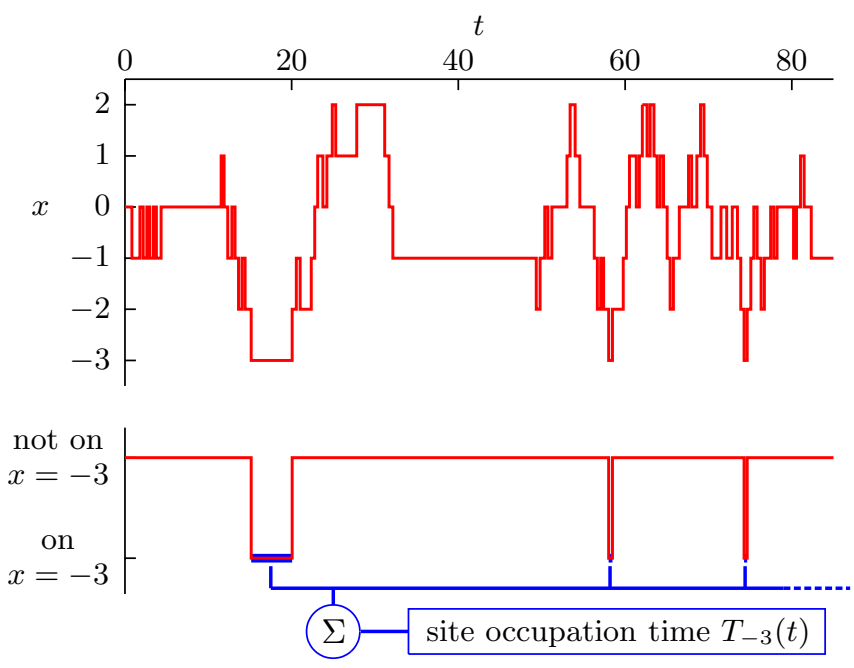

Figure 3. Top: Trajectory of a CTRW with $\alpha=1.5$ in the setting of Fig. 1 Bottom: In order to study the occupation of the lattice site $x=-3$, one can introduce an occupation observable. It alternates between two states, namely the particle is "on $x$ " or "not on $x$." The site occupation time $T_{x}(t)$ is then an accumulation of those waiting times that were spent "on $x$." The stretches of time spent in the "not on $x$ "-state are essentially first-return times of the CTRW.

we will find in Sec. VI that $g_{\mathrm{L}}(\cdot)$ has an infinite norm. Hence, it cannot be treated as a conventional PDF and we call it an infinite density. It is a useful and meaningful object, and contains exactly the statistical information that escapes the bulk scaling analysis.

\section{OCCUPATION TIME STATISTICS}

Let $f\left(t_{x} ; t\right)$ denote the PDF of the occupation time $T_{x}(t)$ and define its double Laplace transform $\widehat{f}(u ; s)=$ $\int_{0}^{\infty} \int_{0}^{\infty} e^{-s t-u t_{x}} f\left(t_{x} ; t\right) d t d t_{x}$. To calculate it, we employ a method developed for the non-ergodic CTRW regime, i.e. $0<\alpha<1$ [11, 12]. It exploits the two-state renewal nature of the occupation process: the site $x$ alternates between being occupied or empty, see Fig. 3. To these two states we can associate sojourn time distributions. The time of continuous occupation of $x$ is distributed as $\psi(\tau)$. The time of continuous absence from $x$ is essentially a first-return time: it measures how long it takes a CTRW particle to return to a site $x$ once it jumped off. We denote its PDF with $\psi_{\mathrm{n} x}(\tau)$ (subscript: "not on $x$ "). Associated Laplace transforms are $\widehat{\psi}(s)=\int_{0}^{\infty} e^{-s \tau} \psi(\tau) d \tau$ and $\widehat{\psi}_{\mathrm{n} x}(s)$ respectively. From these two distributions one can infer the statistics of the occupation time on $x$, see Eq. (A13) of Ref. [12, 44]:

$\widehat{f}(u ; s)=\frac{s \widehat{\psi}_{\mathrm{n} x}(s)[1-\widehat{\psi}(s+u)]+(s+u)\left[1-\widehat{\psi}_{\mathrm{n} x}(s)\right]}{s(s+u)\left[1-\widehat{\psi}(s+u) \widehat{\psi}_{\mathrm{n} x}(s)\right]}$.
We are interested in the large time statistics of occupation times, $t, t_{x} \gg\langle\tau\rangle$. In Laplace space, this corresponds to small $u, s$. For broad-tailed waiting time PDFs as in Eq. (3) with $1<\alpha<2$, the Laplace space asymptotics read

$$
\widehat{\psi}(s) \sim 1-\langle\tau\rangle s+A s^{\alpha}+O\left[s^{2}\right] .
$$

As demonstrated in Ref. [12], one can use subordination arguments in the first-return time analysis to derive the respective small- $s$ expansion for the time of absence from $x$ :

$$
\widehat{\psi}_{\mathrm{n} x}(s) \sim 1-\left\langle\tau_{\mathrm{n} x}^{\mathrm{RW}}\right\rangle\left(\langle\tau\rangle s-A s^{\alpha}\right)+O\left[s^{2}\right],
$$

where $\left\langle\tau_{\mathrm{n} x}^{\mathrm{RW}}\right\rangle$ is the mean first-return time of an ordinary random walk (RW). Here, "ordinary" means that the walker is subject to the same hopping probabilities $q_{x}$, but the waiting times on each lattice site are fixed to unity. The random walks studied in this work are recurrent (i.e. the ultimate return to any $x$ is certain), since they take place under the influence of a binding field $V_{x}$. They also obey the detailed balance condition, Eq. (4). Under these circumstances, we have the simple relation [12, 45, 46]

$$
\left\langle\tau_{\mathrm{n} x}^{\mathrm{RW}}\right\rangle=\frac{1-P_{x}}{P_{x}} .
$$

In order to obtain an approximation for the occupation time PDF in the limit of large times, we now insert the expansions in Eqs. (9) and (10) into the exact Eq. (8); we drop, separately in the numerator and the denominator, anything beyond the two lowest order terms in $s$ and $(s+u)$, respectively. This gives

$$
\widehat{f}(u ; s) \sim \frac{1-C\left[P_{x}(s+u)^{\alpha-1}+\left(1-P_{x}\right) s^{\alpha-1}\right]}{s+u P_{x}-C\left[P_{x}(s+u)^{\alpha}+\left(1-P_{x}\right) s^{\alpha}\right]}
$$

for small, comparable values of $u, s$. The ratio $C=$ $A /\langle\tau\rangle$ quantifies the relative width of the waiting time PDF. By fixing $s$ while expanding at $u \approx 0$, we further get $\widehat{f}(u ; s) \sim s^{-1}+u P_{x} s^{-2}+O\left[u^{2}\right]$. We thus check that the PDF (12) is properly normalized, its mean is indeed the ergodic limit, $\left\langle T_{x}(t)\right\rangle \sim t P_{x}$, and its variance is finite, $\left\langle\left[T_{x}(t)\right]^{2}\right\rangle<\infty$. The latter is natural, since occupation times are bounded, $0 \leq T_{x}(t) \leq t$.

We can now turn our attention to the fluctuations of the occupation times, $\Delta T_{x}(t)$. According to their definition (2), their PDF $g\left(\Delta t_{x} ; t\right)$, is related through $\widehat{g}(k ; s)=$ $\int_{-\infty}^{\infty} \int_{0}^{\infty} e^{-s t+i k \Delta t_{x}} g\left(\Delta t_{x} ; t\right) d t d\left(\Delta t_{x}\right)=\widehat{f}(-i k ; s+$ $\left.i k P_{x}\right)$. Hence,

$$
\begin{aligned}
& \widehat{g}(k ; s) \sim \\
& \frac{1-C\left\{P_{x}\left[s-i k\left(1-P_{x}\right)\right]^{\alpha-1}+\left(1-P_{x}\right)\left(s+i k P_{x}\right)^{\alpha-1}\right\}}{s-C\left\{P_{x}\left[s-i k\left(1-P_{x}\right)\right]^{\alpha}+\left(1-P_{x}\right)\left(s+i k P_{x}\right)^{\alpha}\right\}} .
\end{aligned}
$$

We would like to elaborate some more on the nature of this approximation. We have derived it under the 
premise that we are dealing with long measurement times $t$, hence we took $s$ to be small. Occupation times $T_{x}(t)$ typically grow with time, so we assumed $|k|$ is small and "comparable" to $s$. To be precise, the asymptotic approximation in Eq. (13) is meaningful when $s$ is small and of the same order as $|k|^{\alpha}$, or $|k|$, or anything between. This corresponds exactly to the scaling limits in Eqs. (6) and (7), where the fluctuations $\Delta T_{x}(t)$ are assumed to scale with $t^{1 / \alpha}$ or $t$, respectively. We thus find our heuristic arguments in Sec. III being compatible with Eq. (13). This equation encodes the complete information on the asymptotic distribution of occupation times, but it lives in the abstract Fourier-Laplace space. Additional efforts are required to extract any applicable results. Therefore, we split the problem into two aspects, each focusing on one specific time scaling. This greatly simplifies and clarifies the picture.

\section{THE STABLE LAW OF BULK FLUCTUATIONS}

In order to find the precise bulk scaling function $g_{\mathrm{B}}(\cdot)$, we continue from Eq. (13). The Fourier-Laplace space analogue to the scaling limit (6) is to let $s$ become small, while fixing the ratio $s /|k|^{\alpha}$. Equation (13) by this simplifies and becomes

$$
\begin{aligned}
& \widehat{g}(k ; s) \sim \widehat{g}_{\mathrm{B}}(k ; s)= \\
& \frac{1}{s}\left\{1-C\left[\left(1-P_{x}\right)^{\alpha} P_{x} \frac{(-i k)^{\alpha}}{s}+\left(1-P_{x}\right) P_{x}^{\alpha} \frac{(i k)^{\alpha}}{s}\right]\right\}^{-1}
\end{aligned}
$$

The neglected higher order terms are of the order $O\left[s^{-1 / \alpha}\right]$. To continue, we write

$$
( \pm i k)^{\alpha}=|k|^{\alpha}[\cos (\pi \alpha / 2) \pm i \operatorname{sign}(k) \sin (\pi \alpha / 2)],
$$

choosing principal values for the exponentiation and using $k, \alpha \in \mathbb{R}$. With this, the bulk statistics can be cast into the more compact form

$$
\widehat{g}_{\mathrm{B}}(k ; s)=\frac{1}{s-\kappa^{\alpha} \mid k^{\alpha}[1-i \beta \operatorname{sign}(k) \tan (\alpha \pi / 2)]},
$$

with parameters

$$
\begin{aligned}
& \kappa=\left\{C|\cos (\pi \alpha / 2)|\left[\left(1-P_{x}\right)^{\alpha} P_{x}+\left(1-P_{x}\right) P_{x}^{\alpha}\right]\right\}^{1 / \alpha} \\
& \beta=\frac{\left(1-P_{x}\right)^{\alpha} P_{x}-\left(1-P_{x}\right) P_{x}^{\alpha}}{\left(1-P_{x}\right)^{\alpha} P_{x}+\left(1-P_{x}\right) P_{x}^{\alpha}} .
\end{aligned}
$$

Fourier-Laplace inversion of Eq. (16) yields the soughtafter PDF $g_{\mathrm{B}}\left(\Delta t_{x} ; t\right)$. It is now evident that we can write it in the scaling form

$$
g_{\mathrm{B}}\left(\Delta t_{x} ; t\right) \equiv \frac{1}{t^{1 / \alpha}} g_{\mathrm{B}}\left(\frac{\Delta t_{x}}{t^{1 / \alpha}}\right)=\frac{1}{\kappa t^{1 / \alpha}} \ell_{\alpha, \beta}\left(\frac{\Delta t_{x}}{\kappa t^{1 / \alpha}}\right) .
$$

Thus, at fixed $t$, the scale parameter $\kappa$ measures the bulk PDF's width. For any fixed waiting time parameters, $\kappa$ has a maximum at $P_{x}=1 / 2$ and vanishes if $P_{x}$ tends to either 0 or 1 . Moreover, the characteristic function

$$
\begin{aligned}
\widehat{\ell}_{\alpha, \beta}(k) & =\int_{-\infty}^{\infty} e^{i k y} \ell_{\alpha, \beta}(y) d y \\
& =\exp \left\{-|k|^{\alpha}[1-i \beta \operatorname{sign}(k) \tan (\alpha \pi / 2)]\right\}
\end{aligned}
$$

defines the $\alpha$-stable law [43, 47] $\ell_{\alpha, \beta}(y)$.

Following the intuitive reasoning along the lines of the generalized limit theorem (see Sec. [III), the basic $\alpha$-stable form of the scaling function was to be expected. Nonetheless, some of its specific features are remarkable. The skewness parameter $-1<\beta<1$ indicates an asymmetry; see the sample plots in Fig. 2. This contrasts the familiar Gaussian fluctuations, which appear, e.g. in the ordinary random walk. Also, in other physical systems where Lévy-stable statistics play a role, one encounters typically rather $\beta=0$ or $\beta= \pm 1$. The asymmetry is controlled through $\alpha$ and $P_{x}$. A symmetric distribution is obtained when $P_{x}=1 / 2$, or in the limit $\alpha=2$, which generally recovers Gaussianity.

Another surprising feature of Eq. (17) is its independence of any $P_{x^{\prime}}$ with $x^{\prime} \neq x$. Naturally, at large times, the average occupation time becomes $\left\langle T_{x}(t)\right\rangle \sim t P_{x}$ by virtue of ergodicity. But even more, the complete PDF of fluctuations $\Delta T_{x}(t)$ is controlled by the single steadystate probability $P_{x}$, in conjunction with the waiting time characteristics $\alpha$ and $C$.

One thing appears to be odd though. Because of $0 \leq$ $T_{x}(t) \leq t$, the fluctuations have natural bounds,

$$
-t P_{x} \leq \Delta T_{x}(t) \leq t\left(1-P_{x}\right)
$$

But the support of the stable law $g_{\mathrm{B}}(\cdot)$ is the complete real line. More seriously, $g_{\mathrm{B}}(\cdot)$ takes over the broad tails (3), i.e. $g_{\mathrm{B}}(y) \simeq y^{-\alpha-1}$ for large $y$ [43, 47]. Hence, high-order moments diverge. This includes even the variance, $\left\langle\left[\Delta T_{x}(t)\right]^{2}\right\rangle_{\mathrm{B}}=t^{2 / \alpha} \int_{-\infty}^{\infty} y^{2} g_{\mathrm{B}}(y) d y=\infty$, easily the most common quantitative measure for fluctuations in experimental or numeric data. Evidently, in the case of CTRW, by focusing on the bulk scaling (6), we are missing vital statistical information. To complete the picture, we need to explicitly consider the rare large fluctuations.

\section{THE INFINITE DENSITY OF LARGE FLUCTUATIONS}

The nonstandard scaling limit (7) can be obtained as follows. According to Eq. (18), the largest occupation time fluctuations $\Delta T_{x}(t)$ are of the order of the measurement time $t$ itself. We therefore restart from Eq. (13), let 
again $s$ be small, but now fix the ratio $s /|k|$. This gives

$$
\begin{aligned}
& \widehat{g}(k ; s) \sim \widehat{g}_{\mathrm{L}}(k ; s)=\frac{1}{s}+\frac{C}{s^{2-\alpha}} \times \\
& \quad\left\{P_{x}\left[1-\left(1-P_{x}\right) \frac{i k}{s}\right]^{\alpha}+\left(1-P_{x}\right)\left[1+P_{x} \frac{i k}{s}\right]^{\alpha}\right. \\
& \left.\quad-P_{x}\left[1-\left(1-P_{x}\right) \frac{i k}{s}\right]^{\alpha-1}-\left(1-P_{x}\right)\left[1+P_{x} \frac{i k}{s}\right]^{\alpha-1}\right\} \\
& =\frac{1}{s}+C P_{x} \widehat{R}_{\alpha}\left(\left(1-P_{x}\right) k ; s\right)+C\left(1-P_{x}\right) \widehat{R}_{\alpha}\left(-P_{x} k ; s\right) .
\end{aligned}
$$

In the first step, we dropped all terms which are of $O\left[s^{-1+2(\alpha-1)}\right]$. In the second, we made some simple rearrangements and introduced the auxiliary function

$$
\begin{aligned}
\widehat{R}_{\alpha}(q k ; s) & =\frac{-i k q}{s^{2}}(s-i k q)^{\alpha-1} \\
& =\left[-\frac{i k q}{s}+\frac{(i k q)^{2}}{s^{2}}\right](s-i k q)^{\alpha-2} .
\end{aligned}
$$

We show below that the Fourier-Laplace inversion of $\widehat{R}_{\alpha}$, and hence of Eq. (19) yields the scaling function $g_{\mathrm{L}}(\cdot)$ appearing in Eq. (7).

But before we start, some technical remarks are in place. In Eq. (19), the term $1 / s$ is, strictly speaking, the leading order term in this small- $s$ expansion. It becomes $\delta\left(\Delta t_{x}\right)$ upon Fourier-Laplace inversion, i.e. a Dirac $\delta$-distribution. This does not come as a surprise. We are now rescaling the statistics linearly with time, which is faster than the bulk scaling $\Delta T_{x}(t) \sim t^{1 / \alpha}$, $1<\alpha$. Hence, we "squeeze" the bulk realizations onto the origin. The higher order terms in Eq. (19) contain exactly the information on those fluctuations that deviate from the bulk behavior. Hence, we suppress the $\delta\left(\Delta t_{x}\right)$-term in the oncoming calculations. However, notice that $\delta\left(\Delta t_{x}\right)$ is by itself a PDF. Removing it from the equation naturally causes some issues with normalization for the remaining parts. Therefore, we should not interpret the $g_{\mathrm{L}}(\cdot)$ literally as a PDF. Mathematically, we should rather treat it as a generalized function. When calculating an average with it as $\left\langle\phi\left(\Delta T_{x}(t)\right)\right\rangle_{\mathrm{L}}=$ $\int g_{\mathrm{L}}\left(\Delta t_{x} ; t\right) \phi\left(\Delta t_{x}\right) d\left(\Delta t_{x}\right)$, then we make it act on a test function $\phi$. Not all test functions need to be "suitable", i.e. integrable, with respect to the infinite density. In Sec. VII we elaborate more on this issue.

We now invert the $\widehat{R}_{\alpha}(q k ; s)$ as defined in Eq. (20). We proceed in two steps: first, we go from Laplace space to time, $s \rightarrow t$; second, from Fourier space to fluctuations, $k \rightarrow \Delta t_{x}$. We write the associated transformed functions as $\widetilde{R}_{\alpha}(q k ; s)=\int_{0}^{\infty} e^{-s t} \widetilde{R}_{\alpha}(q k ; t) d t$ and $\widetilde{R}_{\alpha}(q k ; t)=$ $\int_{-\infty}^{\infty} e^{i k \Delta t_{x}} R_{\alpha}\left(\Delta t_{x} / q ; t\right) d\left(\Delta t_{x}\right) / q$. If we start from the second line of Eq. (20), then the first step, the Laplace inversion, $s \rightarrow t$, becomes

$$
\begin{aligned}
\widetilde{R}_{\alpha}(q k ; t)= & -i k q \int_{0}^{t} e^{i k q \omega} \frac{\omega^{-(\alpha-1)}}{\Gamma(2-\alpha)} d \omega \\
& +(i k q)^{2} \int_{0}^{t} e^{i k q \omega}(t-\omega) \frac{\omega^{-(\alpha-1)}}{\Gamma(2-\alpha)} d \omega .
\end{aligned}
$$

We can put this in a more elegant form, which makes explicit the time scaling nature of the function:

$$
\widetilde{R}_{\alpha}(q k ; t)=\frac{\alpha}{t^{\alpha-1}|\Gamma(1-\alpha)|} \widetilde{\mathcal{I}}(\zeta), \quad \zeta=k q t,
$$

where

$$
\begin{aligned}
\widetilde{\mathcal{I}}(\zeta)= & \frac{1}{\alpha(\alpha-1)}\left[-i \zeta \int_{0}^{1} e^{i \zeta \omega} \omega^{-(\alpha-1)} d \omega\right. \\
& \left.+(i \zeta)^{2} \int_{0}^{1} e^{i \zeta \omega}(1-\omega) \omega^{-(\alpha-1)} d \omega\right] .
\end{aligned}
$$

The remaining task is the Fourier inversion $k \rightarrow \Delta t_{x}$, or respectively, $\zeta \rightarrow z=\Delta t_{x} /(q t)$. For this, we translate powers of $\zeta$ into derivatives $\partial / \partial z$ and the exponential $e^{i \zeta \omega}$ into $\delta(z-\omega)$. We thus get the inverse Fourier-Transform of $\widetilde{\mathcal{I}}(\zeta)$,

$$
\begin{gathered}
\mathcal{I}(z)=\frac{1}{\alpha(\alpha-1)}\left\{\frac{\partial}{\partial z} \int_{0}^{1} \delta(z-\omega) \omega^{-(\alpha-1)} d \omega\right. \\
\left.+\frac{\partial^{2}}{\partial z^{2}} \int_{0}^{1} \delta(z-\omega)\left[(1-\omega) \omega^{-(\alpha-1)}\right] d \omega\right\} \\
=\frac{1}{\alpha(\alpha-1)}\left\{\frac{\partial}{\partial z}\left[\mathbf{1}\{0<z \leq 1\} z^{-(\alpha-1)}\right]\right. \\
\left.+\frac{\partial^{2}}{\partial z^{2}}\left[\mathbf{1}\{0<z \leq 1\}(1-z) z^{-(\alpha-1)}\right]\right\} .
\end{gathered}
$$

The indicator function $\mathbf{1}\{\cdot\}$ is 1 when the condition in the argument is fulfilled, and 0 otherwise. Hence, it is a discontinuous step function, and its differentiation gives rise to several $\delta$-distributions in the expression. For reasons that we elaborated on above, we drop the contributions from the boundary $z=0$. In App. $\mathrm{A}$, we show that the other peaks at $z=1$ have zero contribution. Performing the differentiations in Eq. (24) therefore gives

$$
\mathcal{I}(z)=\mathbf{1}\{0<z \leq 1\} z^{-1-\alpha}\left(1-\frac{\alpha-1}{\alpha} z\right) .
$$

Tracing back the definitions in Eqs. (23), (22) and (19), we finally arrive at

$$
\begin{gathered}
g_{\mathrm{L}}\left(\Delta t_{x} ; t\right) \equiv \frac{1}{t^{\alpha}} g_{\mathrm{L}}\left(\frac{\Delta t_{x}}{t}\right)=\frac{C \alpha}{t^{\alpha}|\Gamma(1-\alpha)|} \times \\
\begin{cases}\frac{P_{x}}{1-P_{x}} \mathcal{I}\left(\frac{\Delta t_{x}}{t\left(1-P_{x}\right)}\right), & 0<\frac{\Delta t_{x}}{t} \leq 1-P_{x}, \\
\frac{1-P_{x}}{P_{x}} \mathcal{I}\left(\frac{\left|\Delta t_{x}\right|}{t P_{x}}\right), & -P_{x} \leq \frac{\Delta t_{x}}{t}<0, \\
0, & \text { otherwise. }\end{cases}
\end{gathered}
$$


This scaling function is defined within the appropriate bounds (18) and correctly predicts the behavior of the large occupation times, see Fig. 2. Again, the expression depends solely on $\alpha, C$, and the single equilibrium probability $P_{x}$, and it is symmetric only if $P_{x}=1 / 2$.

Due to the fast divergence $\simeq\left|\Delta t_{x}\right|^{-\alpha-1}$ at the origin, $g_{\mathrm{L}}(\cdot)$ is not normalizable. This is why we call it an infinite density. This distinguished property should not be a cause of concern. Of course, at any finite time $t$, the exact PDF $g(\cdot)$ is perfectly normalized. Its central part is best approximated by the stable law $g_{\mathrm{B}}(\cdot)$, Eq. (17), while large fluctuations are best studied in terms of $g_{\mathrm{L}}(\cdot)$, Eq. (25). In particular, the pole of the infinite density is completely congruent with the tails of the $\alpha$-stable bulk distribution [47]: Eqs. (6), (7), (17), and (25) consistently yield

$$
\begin{aligned}
g\left(\Delta t_{x} ; t\right) \sim & \frac{1}{t^{1 / \alpha}} g_{\mathrm{B}}\left(\frac{\Delta t_{x}}{t^{1 / \alpha}}\right) \sim \frac{1}{t^{\alpha}} g_{\mathrm{L}}\left(\frac{\Delta t_{x}}{t}\right) \\
\sim & \frac{C \alpha\left(1-P_{x}\right)^{\alpha} P_{x} t}{|\Gamma(1-\alpha)|} \Delta t_{x}-\alpha-1 \\
& \text { for } \kappa t^{1 / \alpha} \ll \Delta t_{x} \ll t\left(1-P_{x}\right), \\
g\left(\Delta t_{x} ; t\right) \sim & \frac{1}{t^{1 / \alpha}} g_{\mathrm{B}}\left(\frac{\Delta t_{x}}{t^{1 / \alpha}}\right) \sim \frac{1}{t^{\alpha}} g_{\mathrm{L}}\left(\frac{\Delta t_{x}}{t}\right) \\
\sim & \frac{C \alpha\left(1-P_{x}\right) P_{x}^{\alpha} t}{|\Gamma(1-\alpha)|}\left|\Delta t_{x}\right|^{-\alpha-1} \\
& \text { for }-t P_{x} \ll \Delta t_{x} \ll-\kappa t^{1 / \alpha} .
\end{aligned}
$$

The power law $\left|\Delta t_{x}\right|^{-\alpha-1}$ is responsible for both types of unphysical divergences in the asymptotics: the divergent moments of the stable laws (17) and the divergent norm of the infinite density (25). But by patching the two asymptotic expressions along the common power law overlap we obtain a complete, consistent statistical longtime description, bare of any such dubious divergences.

\section{ENSEMBLE AVERAGES}

When calculating an average of the form $\left\langle\phi\left(\Delta T_{x}(t)\right)\right\rangle$, we are confronted with the question of how to apply the above asymptotic results. Is the average obtained from the Lévy-stable bulk statistics $g_{\mathrm{B}}(\cdot)$, Eq. (17)? Or is it sensitive to the large fluctuations, so that we need the infinite density $g_{\mathrm{L}}(\cdot)$, Eq. (25)? Or may we even have to consider the complete, yet implicit solution $\widehat{g}(\cdot)$, Eq. (13)? In general, we find that $g_{\mathrm{B}}(\cdot)$ and $g_{\mathrm{L}}(\cdot)$ complement one another and yield the entire information on the long-time asymptotics. This becomes most evident in the study of absolute $q$ th order moments, $\phi\left(\Delta T_{x}(t)\right)=\left|\Delta T_{x}(t)\right|^{q}$. We show in App. B that, with the exception of $q=\alpha$, the moments fall into two distinct classes, which are exclusively integrable with respect to either the stable law or the infinite density:

$$
\left\langle\left|\Delta T_{x}(t)\right|^{q}\right\rangle \sim \begin{cases}\left\langle\left|\Delta T_{x}(t)\right|^{q}\right\rangle_{\mathrm{B}}=M_{q}^{<} t^{q / \alpha}, & q<\alpha, \\ \left\langle\left|\Delta T_{x}(t)\right|^{q}\right\rangle_{\mathrm{L}}=M_{q}^{>} t^{q+1-\alpha}, & q>\alpha,\end{cases}
$$

where

$$
\begin{aligned}
M_{q}^{<} & =\kappa^{q} \int_{-\infty}^{\infty}|y|^{q} \ell_{\alpha, \beta}(y) d y \quad \text { [see Ref. [47], chap. 1.2], } \\
M_{q}^{>} & =\frac{C \alpha\left[P_{x}\left(1-P_{x}\right)^{q}+\left(1-P_{x}\right) P_{x}^{q}\right]}{|\Gamma(1-\alpha)|} \int_{0}^{1} z^{q} \mathcal{I}(z) d z \\
& =\frac{C q\left[P_{x}\left(1-P_{x}\right)^{q}+\left(1-P_{x}\right) P_{x}^{q}\right]}{|\Gamma(1-\alpha)|(q-\alpha)(q-\alpha+1)} .
\end{aligned}
$$

We stress that the coefficients $M_{q}^{<}$and $M_{q}^{>}$are finite in their respective parameter domains. The stable laws $\ell_{\alpha, \beta}(y)$ do possess finite moments of order $q<\alpha$. The expression $z^{q} \mathcal{I}(z)$ is perfectly integrable when $q>\alpha$. We can thus use, in this context, the infinite density $g_{\mathrm{L}}(\cdot)$ as if it was an ordinary PDF - despite the pole at the origin. We add that the borderline case $\left\langle\left|\Delta T_{x}(t)\right|^{\alpha}\right\rangle \simeq$ $t \ln t$ is obtained by carefully accounting for contributions from both the bulk and large fluctuations, see App. B.

According to Eq. (27a), the different time scalings of bulk versus large fluctuations manifest in a dual scaling of moments. The same duality has been discussed with respect to strong anomalous diffusion 37, 48. In particular, we have $\left\langle\left[\Delta T_{x}(t)\right]^{2}\right\rangle \sim\left\langle\left[\Delta T_{x}(t)\right]^{2}\right\rangle_{\mathrm{L}} \simeq t^{3-\alpha}$. Even the variance - usually not perceived as a high-order moment - does probe the statistics of the large fluctuations. Its increase with time is faster than anticipated from the bulk scaling $\left(\left[\Delta T_{x}(t)\right]^{2} \sim t^{2 / \alpha}\right)$.

\section{TIME-AVERAGED OBSERVABLES}

In this section, we show that the connection between dual scaling laws and stable and infinite densities is not specific to occupation time statistics, but extends to a larger class of observables. Assume an observable $\mathcal{O}$ takes on the value $o_{x}$ when the random walker sits on site $x$. Then, by virtue of ergodicity, Eq. (1), the time average

$$
\overline{\mathcal{O}}(t)=\frac{1}{t} \sum_{x} o_{x} T_{x}(t)
$$

converges to the equilibrium ensemble average

$$
\langle\mathcal{O}\rangle_{\mathrm{eq}}=\sum_{x} o_{x} P_{x}
$$

in the limit $t \rightarrow \infty$. At finite time $t$, we define the fluctuations of the time average as

$$
\overline{\Delta \mathcal{O}}(t)=\overline{\mathcal{O}}(t)-\langle\mathcal{O}\rangle_{\mathrm{eq}}=\frac{1}{t} \sum_{x} o_{x} \Delta T_{x}(t)
$$

We denote the respective $\mathrm{PDF}$ by $h(\overline{\Delta o} ; t)$. For reasons which become apparent below, we restrict ourselves to 
observables and systems where the equilibrium average $\left\langle|\Delta \mathcal{O}|^{\alpha}\right\rangle_{\text {eq }}=\sum_{x}\left|o_{x}-\langle\mathcal{O}\rangle_{\text {eq }}\right|^{\alpha} P_{x}$ is finite.

The general time average is defined through Eq. (28) in terms of the occupation time fractions $T_{x}(t) / t$. Conversely, the occupation time fraction of a specific region $\mathcal{D}$ of the lattice is proportional to the time average of a particular observable. Namely, we can define an occupation observable $\mathcal{O}$ through an indicator function $\mathbf{1}\{\cdot\}$ :

$$
o_{x}=\mathbf{1}\{x \in \mathcal{D}\}= \begin{cases}1 & \text { for } x \in \mathcal{D}, \\ 0 & \text { for } x \notin \mathcal{D} .\end{cases}
$$

The time average of an occupation observable can be written as

$$
\overline{\mathcal{O}}(t)=\frac{1}{t} \sum_{x} \mathbf{1}\{x \in \mathcal{D}\} T_{x}(t)=\frac{1}{t} \sum_{x \in \mathcal{D}} T_{x}(t)=\frac{T_{\mathcal{D}}(t)}{t},
$$

and likewise

$$
\overline{\Delta \mathcal{O}}(t)=\frac{\Delta T_{\mathcal{D}}(t)}{t} .
$$

In the simplest case, $\mathcal{D}$ would only consist of a single lattice point $x$ (see, e.g., Fig. 3, where $x=-3$ ). We then return to the scenario of the previous sections. In this sense, the single-site occupation time fractions $T_{x}(t) / t$ are special cases of the general time average $\overline{\mathcal{O}}(t)$ in Eq. (28). We are hence now extending the discussion to a broader class of observables. The detailed calculus is similar to the previous and can be found in App. [C] We focus on the implied generalizations for the dual time scaling, the bulk and the large fluctuation laws.

\section{A. The dual time scaling of time averages}

The fluctuations of a general time average $\overline{\Delta \mathcal{O}}(t)$ should, according to definition (30), have essentially the same time scaling behavior as the fluctuations of the occupation time fractions $\Delta T_{x}(t) / t$. That is, for bulk fluctuations we expect that $\overline{\Delta \mathcal{O}}(t) \sim t^{1 / \alpha} / t=t^{-(\alpha-1) / \alpha}$, while the large fluctuations should be of constant order. Indeed, we find the bulk behavior

$$
h(\overline{\Delta o} ; t) \sim t^{(\alpha-1) / \alpha} h_{\mathrm{B}}\left(\overline{\Delta o} t^{(\alpha-1) / \alpha}\right),
$$

and the large fluctuations scaling limit is

$$
h(\overline{\Delta o} ; t) \sim \frac{1}{t^{\alpha-1}} h_{\mathrm{L}}(\overline{\Delta o}) .
$$

Equations (6) and (7) are to be viewed as special cases. This basic nature of the dual time scaling is thus universal for all time averages, including occupation times. What remains to be investigated is the exact form of the scaling functions $h_{\mathrm{B}}(\cdot)$ and $h_{\mathrm{L}}(\cdot)$ and their sensitivity to the choice of the observable $\mathcal{O}$.

\section{B. The stable law of bulk fluctuations}

The bulk scaling limit for large $t$ reads

$$
\begin{aligned}
h_{\mathrm{B}}(\overline{\Delta o} ; t) & \equiv t^{(\alpha-1) / \alpha} h_{\mathrm{B}}\left(\overline{\Delta o} t^{(\alpha-1) / \alpha}\right) \\
& =\frac{t^{(\alpha-1) / \alpha}}{\kappa} \ell_{\alpha, \beta}\left(\frac{\overline{\Delta o} t^{(\alpha-1) / \alpha}}{\kappa}\right),
\end{aligned}
$$

where now

$$
\kappa=\left[C|\cos (\pi \alpha / 2)|\left\langle|\Delta \mathcal{O}|^{\alpha}\right\rangle_{\mathrm{eq}}\right]^{1 / \alpha}
$$

and the $\alpha$-stable law $\ell_{\alpha, \beta}(y)$ is as in Eq. (17d), only with $\beta$ defined as

$$
\beta=\frac{\left\langle|\Delta \mathcal{O}|^{\alpha}\right\rangle_{\mathrm{eq}, \Delta \mathcal{O}>0}-\left\langle|\Delta \mathcal{O}|^{\alpha}\right\rangle_{\mathrm{eq}, \Delta \mathcal{O}<0}}{\left\langle|\Delta \mathcal{O}|^{\alpha}\right\rangle_{\mathrm{eq}}} .
$$

We have introduced here the $\alpha$ th order absolute moments of equilibrium fluctuations

$$
\begin{aligned}
\left\langle|\Delta \mathcal{O}|^{\alpha}\right\rangle_{\mathrm{eq}} & =\left\langle\left|\mathcal{O}-\langle\mathcal{O}\rangle_{\mathrm{eq}}\right|^{\alpha}\right\rangle_{\mathrm{eq}} \\
& =\sum_{x}\left|o_{x}-\langle\mathcal{O}\rangle_{\mathrm{eq}}\right|^{\alpha} P_{x}
\end{aligned}
$$

and respective constrained averages

$$
\begin{aligned}
\left\langle|\Delta \mathcal{O}|^{\alpha}\right\rangle_{\text {eq, }, \Delta \mathcal{O} \gtrless z} & =\left\langle\mathbf{1}\left\{\mathcal{O}-\langle\mathcal{O}\rangle_{\text {eq }} \gtrless z\right\}\left|\mathcal{O}-\langle\mathcal{O}\rangle_{\text {eq }}\right|^{\alpha}\right\rangle_{\text {eq }} \\
& =\sum_{x}{ }^{\gtrless}\left|o_{x}-\langle\mathcal{O}\rangle_{\text {eq }}\right|{ }^{\alpha} P_{x} .
\end{aligned}
$$

The constrained sums $\sum_{x}^{\gtrless}$ run over all $x$ where $o_{x} \gtrless$ $z+\langle\mathcal{O}\rangle_{\text {eq }}$, respectively.

The main observation here is that the basic $\alpha$-stable character of bulk fluctuations is universal for all observables. Still, the details are observable-specific, in particular the skewness parameter $\beta$. If the observable $\mathcal{O}$ in equilibrium symmetrically fluctuates around its mean, then $\beta=0$, so the fluctuations of the time average are also symmetric. The converse, however, does not hold. One can think of a situation where $\mathcal{O}$ is asymmetric in equilibrium, but in such a way that we happen to have $\left\langle|\Delta \mathcal{O}|^{\alpha}\right\rangle_{\text {eq }, \Delta \mathcal{O}<0}=\left\langle|\Delta \mathcal{O}|^{\alpha}\right\rangle_{\text {eq, }, \Delta \mathcal{O}>0}$, so that the time average is symmetric. Thus, there seems to be no simple one-to-one correspondence between the symmetry of an observable in equilibrium and the symmetry of the respective time average - that is, with respect to the bulk statistics. 


\section{The infinite density of large fluctuations}

The scaling function in the large fluctuations limit (33) is given through

$$
\begin{aligned}
h_{\mathrm{L}}(\overline{\Delta o} ; t) & \equiv \frac{1}{t^{\alpha-1}} h_{\mathrm{L}}(\overline{\Delta o}) \\
& =\frac{C \alpha}{t^{\alpha-1}|\Gamma(1-\alpha)|} \begin{cases}\mathcal{I}_{\mathcal{O}}^{>}(\overline{\Delta o}), & 0<\overline{\Delta o}, \\
\mathcal{I}_{\mathcal{O}}^{<}(\overline{\Delta o}), & \overline{\Delta o}<0,\end{cases}
\end{aligned}
$$

introducing the infinite densities

$$
\begin{aligned}
\mathcal{I}_{\mathcal{O}}^{\gtrless}(z)=|z|^{-\alpha-1}\left[\left\langle|\Delta \mathcal{O}|^{\alpha}\right\rangle_{\mathrm{eq}}, \Delta \mathcal{O} \gtrless z\right. \\
- \\
\left.-\frac{\alpha-1}{\alpha}|z|\left\langle|\Delta \mathcal{O}|^{\alpha-1}\right\rangle_{\mathrm{eq}, \Delta \mathcal{O} \gtrless z}\right] .
\end{aligned}
$$

The non-integrable pole $\simeq|\overline{\Delta o}|^{-\alpha-1}$ at the origin consistently overlaps with the broad tails of the $\alpha$-stable bulk PDF in Eq. (34). Therefore, this feature is again universal for all observables. But the specifics of the observable do determine the shape of the complete infinite density. We stress that the constrained moments that enter Eq. (36) explicitly depend on $\overline{\Delta o}$. Because of this, the analysis of the large fluctuations does reveal the symmetry and other characteristics of the observable $\mathcal{O}$. In contrast to the mere bulk statistics, the PDF $h_{\mathrm{L}}(\cdot)$ is symmetric if and only if the $\mathcal{O}$ fluctuates symmetrically around its mean in the equilibrium state.

\section{Example: Time-averaged position of a particle biased towards a reflective wall}

As an example, we study the time-averaged position,

$$
o_{x}=x, \quad \overline{\mathcal{O}}(t)=\bar{X}(t),
$$

for a thermal CTRW as defined in Sec. II] We consider a constant force $-F<0$ as a bias, which drives the particle against a reflective wall at $x=0$. For convenience, we define a parameter $\mu=F /\left(k_{\mathrm{B}} \mathcal{T}\right)$ that quantifies the competition between the localizing bias $F$ and the diffusive spreading at finite temperature $\mathcal{T}$. On our lattice we define the discrete potential landscape

$$
\frac{V_{x}}{k_{\mathrm{B}} \mathcal{T}}= \begin{cases}\infty, & x \leq-1 \\ \ln \left(1+e^{\mu}\right), & x=0 \\ \mu x, & x \geq 1\end{cases}
$$

which is linear in $x$ for all $x \geq 1$. Thermal detailed balance, Eq. (4), then implies that

$$
q_{x}= \begin{cases}1, & x=0 \\ \frac{1}{1+e^{\mu}} \equiv q, & x \geq 1\end{cases}
$$

Hence, all steps are taken preferably to the left with probability $1-q>1 / 2$; only at the origin, they are reflected to the right. The Boltzmann-Gibbs equilibrium PDF, Eq. (5), for these dynamics reads

$$
P_{x}=\frac{1}{Z} \begin{cases}0, & x \leq-1, \\ \frac{1}{1+e^{\mu}}, & x=0, \\ e^{-\mu x}, & x \geq 1,\end{cases}
$$

with the partition function $Z=[\sinh (\mu)]^{-1}$. The equilibrium position is given by

$$
\langle X\rangle_{\mathrm{eq}}=\sum_{x=0}^{\infty} x P_{x}=\frac{e^{\mu}+1}{\left(e^{\mu}-1\right)^{2}} .
$$

For the statistics of the time average, we further need the absolute moments of Eq. (35):

$$
\left\langle|\Delta X|^{\alpha}\right\rangle_{\mathrm{eq}, \Delta X>z}=\sum_{x=\left\lceil\langle X\rangle_{\mathrm{eq}}+z\right\rceil}^{\infty}\left(x-\langle X\rangle_{\mathrm{eq}}\right)^{\alpha} \frac{e^{-\mu x}}{Z}
$$

for all $z>0$. We used the notation $\lceil x\rceil$ to designate the smallest integer $\geq x$. Similarly, we can find, for all $-\langle X\rangle_{\mathrm{eq}} \leq z<0$

$$
\left\langle|\Delta X|^{\alpha}\right\rangle_{\mathrm{eq}, \Delta X<z}=\sum_{x=0}^{\left\lfloor\langle X\rangle_{\mathrm{eq}}+z\right\rfloor}\left(\langle X\rangle_{\mathrm{eq}}-x\right)^{\alpha} \frac{e^{-\mu x}}{Z} .
$$

Here, $\lfloor x\rfloor$ is the largest integer $\leq x$. Of course we implicitly obtain

$$
\left\langle|\Delta X|^{\alpha}\right\rangle_{\mathrm{eq}}=\left\langle|\Delta X|^{\alpha}\right\rangle_{\mathrm{eq}, \Delta X<0}+\left\langle|\Delta X|^{\alpha}\right\rangle_{\mathrm{eq}, \Delta X>0} .
$$

Equations (41) and (42) contain all the equilibrium statistics that we need to calculate the asymptotic distribution of the time-averaged position $\bar{X}(t)$. Its ensemble mean falls onto the equilibrium mean, $\langle\bar{X}(t)\rangle \sim\langle X\rangle_{\mathrm{eq}}$. The bulk PDF of the fluctuations $\overline{\Delta X}(t)$ is given through Eq. (32), where we insert the equilibrium quantities in Eqs. (41) and (42). Plots are provided in Fig. 4, left panel, for different values of the bias strength $\mu$. When the bias is stronger ( $\mu$ larger), then the PDF of the fluctuations gets more peaked ( $\kappa$ decreases) and more symmetric $(\beta>0$ decreases $)$.

The large fluctuations scaling limit is obtained by using Eqs. (41) and (42) in Eq. (33). The respective plots can be found in the right panel of Fig. 4. In contrast to the universal Lévy-stable bulk statistics, the structure of the emergent infinite density does reveal two defining properties of the underlying CTRW. First, the reflective boundary condition causes the fluctuations to be bounded from the left, $-\langle X\rangle_{\text {eq }} \leq \overline{\Delta X}(t)$. Second, the infinite density exhibits discontinuous steps at points where $\langle X\rangle_{\mathrm{eq}}+\overline{\Delta x}$ becomes an integer, an obvious signature of the discrete lattice structure. The effect is most pronounced for a strong bias, since then the CTRW samples only a small number of lattice sites. 

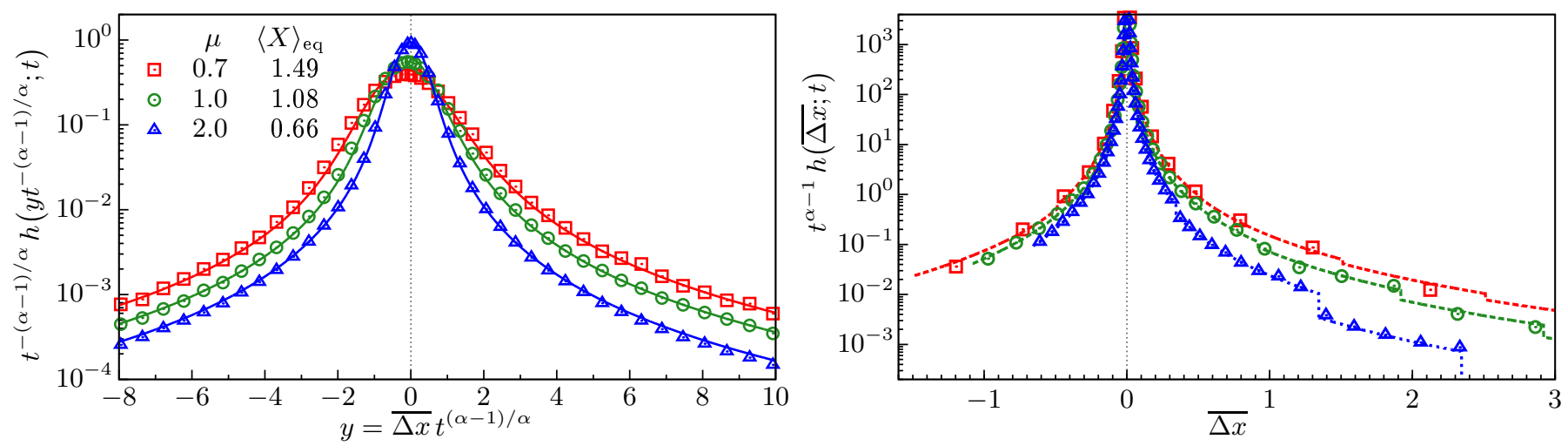

Figure 4. Scaled PDF of fluctuations $\overline{\Delta X}(t)$ of the time-averaged position for confined CTRW. The confinement is a constant bias to the left, $V_{x} /\left(k_{\mathrm{B}} \mathcal{T}\right)=\mu x$, towards a reflecting boundary at the origin. Different symbols are simulation results for different bias strengths, $\mu=0.7\left(7 \times 10^{5}\right.$ trajectories, $\left.t=10^{7}\right), \mu=1.0\left(6 \times 10^{6}\right.$ trajectories, $\left.t=10^{6}\right)$ and $\mu=2.0\left(2 \times 10^{7}\right.$ trajectories, $\left.t=10^{5}\right)$. In all cases, waiting times were broad-tailed as in Eq. (3) with $\alpha=1.5,\langle\tau\rangle=1$ and $A=0.5$. Both panels depict the same data. Left: Scaling with $t^{-(\alpha-1) / \alpha}$ as in Eq. (32) yields the asymmetric, stable bulk statistics $h_{\mathrm{B}}(\cdot)$ of Eq. (34) (continuous lines), with the equilibrium statistics given by Eqs. (41) and (42). Right: The nonstandard time scaling in Eq. (33) produces the infinite density $h_{\mathrm{L}}(\cdot)$ for the large fluctuations as in Eq. (36) (dashed lines). The infinite density is bounded only to the left due to the reflective wall, and its discontinuous steps reveal the discrete lattice structure. This information could not be extracted from the bulk plot on the left.

\section{SUMMARY AND OUTLOOK}

Within the framework of CTRW, we studied bounded, ergodic dynamics close to the non-ergodic phase. In this regime, equilibrium statistical mechanics, in particular the Boltzmann-Gibbs laws, dictate the values of occupation times and time averages in the limit of infinite measurement times. But at finite times, the fluctuations of these quantities are significant and are to be analyzed on the footing of dual scaling laws, such as Eqs. (6)-(7) or (32)-33). Bulk fluctuations are to be distinguished from the rare, large fluctuations. The two emergent asymptotic scaling laws complement one another. Thus, the behavior of physical observables, like the $q$ th order moments considered in Eqs. (27), are to be derived from bulk or large fluctuations, or both.

The bulk fluctuations are asymptotically distributed according to asymmetric Lévy-stable laws. This qualitative feature is universal for occupation times and general time averages alike, see Eq. (17) or (34). Only a single parameter, the skewness $\beta$, depends to some extent on the binding potential and the observable of interest. The rare large fluctuations also exhibit universality, in so far as they are generally described in terms of infinite densities, see Eq. (25) or (36). But the precise analytic form of the infinite density is much more sensitive to the details of the system and the particular observable under study. For instance, the natural bounds of the occupation times or time averages are only probed by the large fluctuations; also the discrete/continuous structure of the phase space is only reflected in the infinite density behavior.

Our equations connect the emergent Lévy-stable laws and infinite densities with the occupation probability $P_{x}$ of an equilibrium ensemble of independent random walkers. In a thermal system, we can thus relate the Boltzmann-Gibbs equilibrium statistics with the finitetime fluctuations around the ergodic limit. In the case of occupation times, this relationship is remarkably simple. For a given lattice site $x$, the single equilibrium occupation probability $P_{x}$ controls the asymptotic distribution of both bulk and large fluctuations. Beyond that, only two dynamical parameters determine the distribution: the tail exponent $\alpha$ and the relative width $C=A /\langle\tau\rangle$ of the waiting time distribution (3).

Hence, the equilibrium laws of statistical mechanics have a strong connection with asymmetric Lévy-stable laws and infinite densities. We believe that similar relationships can be found in a vast variety of systems beyond CTRW. They derive from the broad sojourn time distributions with power law tails as in Eq. (3). This characteristic also appears in stochastic [10, 14] and deterministic [30 33, 42] models of complex motion, and has been measured experimentally [15, 18-20]. While the detailed asymptotic statistics of these systems might be complicated, our dual scaling approach may prove to be an effective analytical tool. Experimental studies of such systems may reveal the connection between model parameters $\left(\alpha, C, P_{x}\right)$ and other measurable quantities (temperature, particle size, confinement characteristics, etc.). An ambitious, yet desirable long-term goal is to embed the limit laws found here into large deviations theory [49]. Furthermore, aging effects [4, 38] should be taken into consideration, if the CTRW-like process has been initiated long before we start to measure occupation times or time averages. We expect aging to be relevant in particular for the infinite density of large fluctuations. The regime $\alpha>2$ is an alternative direction to extend our studies. We believe that, in this case, an infinite den- 
sity still provides the large fluctuation statistics, due to the divergence of moments $\left\langle\tau^{k}\right\rangle$ with $k>\alpha$. But how rare these large fluctuations are, and how their infinite density looks like or connects to the presumably Gaussian bulk distribution, are outstanding questions.

\section{ACKNOWLEDGMENTS}

We acknowledge funding from the Israel Science Foundation.

\section{Appendix A: Peaked contributions to the infinite density $\mathcal{I}(z)$ in Eq. (24)}

The considerations that we expressed at the beginning of Sec. VI have led us to exercise care when treating the outer boundaries $z=1$ in Eq. (24), and respectively $\Delta t_{x}=-P_{x} t,\left(1-P_{x}\right) t$ in Eq. (25). Generally, derivatives of the step function $\mathbf{1}\{z \leq 1\}$ lead to peaks of the form $\delta(z-1)$. But for the CTRWs we studied in this work, such contributions are not present, as we show now. We abbreviate the expression $z^{-(\alpha-1)}$ appearing in Eq. (24) with $F(z)$ and write down the distributional identity

$$
\left\{\frac{\partial}{\partial z}[\mathbf{1}\{z \leq 1\} F(z)]\right\}=\mathbf{1}\{z \leq 1\} F^{\prime}(z)-\delta(z-1) F(z) .
$$

In principle, $F(z)$ could be any sufficiently well-behaved conventional function, which in particular does not have a pole at $z=1$. Similarly, we can write

$$
\begin{aligned}
& \left\{\frac{\partial^{2}}{\partial z^{2}}[\mathbf{1}\{z \leq 1\} F(z)]\right\}= \\
& \quad=\mathbf{1}\{z \leq 1\} F^{\prime \prime}(z)-2 \delta(z-1) F^{\prime}(z)+\delta^{\prime}(z-1) F(z) \\
& \quad=\mathbf{1}\{z \leq 1\} F^{\prime \prime}(z)-\delta(z-1) F^{\prime}(z)+\delta(z-1) F(z) \frac{\partial}{\partial z} .
\end{aligned}
$$

The derivative on the right-hand side acts upon any subjected test function. With respect to Eq. (24), the above relation is useful for evaluating the expression containing $F(z)=(1-z) z^{-(\alpha-1)}$.

With the identities (A1), we can continue Eq. (24) as

$$
\begin{aligned}
\mathcal{I}(z)= & \frac{\mathbf{1}\{0<z \leq 1\}}{\alpha(\alpha-1)}\left\{-(\alpha-1) z^{-\alpha}+\right. \\
& \left.\quad+[(\alpha-1) \alpha+(2-\alpha)(\alpha-1) z] z^{-\alpha-1}\right\} \\
& -\frac{\delta(z-1)}{\alpha(\alpha-1)}\left\{z^{-(\alpha-1)}-[\alpha-1+(2-\alpha) z] z^{-\alpha}\right\} \\
& +\frac{\delta(z-1)}{\alpha(\alpha-1)}\left\{(1-z) z^{-(\alpha-1)}\right\} \frac{\partial}{\partial z} \\
= & \mathbf{1}\{0<z \leq 1\} z^{-1-\alpha}\left(1-\frac{\alpha-1}{\alpha} z\right) .
\end{aligned}
$$

We observe that the $\delta$-peaks at the boundary $z=1$ indeed have zero contribution, and Eq. (25a) holds true.

\section{Appendix B: Absolute moments of all orders, Eq. (27)}

We know that at large times $t$, the central part of the PDF $g\left(\Delta t_{x} ; t\right)$ is well approximated by $g_{\mathrm{B}}\left(\Delta t_{x} ; t\right)$, while the outer parts are close to $g_{\mathrm{L}}\left(\Delta t_{x} ; t\right)$. Inside two intermediate overlap regions, both approximations feature the power law behavior given in Eq. (26). We now introduce a positive, monotonic function $c(t)$, such that $c(t)$ and $-c(t)$ are bound to lie inside the positive and negative power law regions, respectively, i.e.

$-t P_{x} \ll-c(t) \ll-\kappa t^{1 / \alpha} \wedge \kappa t^{1 / \alpha} \ll c(t) \ll t\left(1-P_{x}\right)$.

Notice that the distance between the boundaries broadens as time increases. Therefore, we can always find a function $c(t)$ that remains inside this region, at least beyond some finite threshold time. We even have some freedom in defining its time dependence: $c(t) \simeq t^{1 / \alpha}$ is fine, or $c(t) \simeq t$, or anything in between. We make use of this fact below.

With the function $c(t)$ we can divide the value of an absolute moment into two contributions:

$$
\begin{aligned}
\left\langle\left|\Delta T_{x}(t)\right|^{q}\right\rangle & =\left(\int_{\left|\Delta t_{x}\right| \leq c(t)}+\int_{\left|\Delta t_{x}\right|>c(t)}\right)\left|\Delta t_{x}\right|^{q} g\left(\Delta t_{x} ; t\right) d\left(\Delta t_{x}\right) \\
& =t^{q / \alpha} \int_{|y| \leq c(t) / t^{1 / \alpha}}|y|^{q} t^{1 / \alpha} g\left(y t^{1 / \alpha} ; t\right) d y+t^{-(\alpha-1)+q} \int_{|z|>c(t) / t}|z|^{q} t^{\alpha} g(z t ; t) d z \\
& \sim t^{q / \alpha} \int_{|y| \leq c(t) / t^{1 / \alpha}}|y|^{q} g_{\mathrm{B}}(y) d y+t^{-(\alpha-1)+q} \int_{|z|>c(t) / t}|z|^{q} g_{\mathrm{L}}(z) d z
\end{aligned}
$$

On the last line, we used the asymptotic expressions as in Eqs. (6) and (7). This is allowed, since the boundary $c(t)$ asserts that both integrals operate on regions where the respective approximation becomes more and more accurate 
with time. We now treat the three classes of moments separately.

\section{Low-order moments, $0<q<\alpha$}

We choose $c(t) \equiv t c_{1}$, with some $0<c_{1} \ll \min \left\{P_{x}, 1-P_{x}\right\}$, and continue Eq. (B21):

$$
\begin{array}{rlrl}
\left\langle\left|\Delta T_{x}(t)\right|^{q}\right\rangle & \sim t^{q / \alpha} \int_{|y| \leq t^{1-1 / \alpha} c_{1}}|y|^{q} g_{\mathrm{B}}(y) d y & +t^{-(\alpha-1)+q} \int_{|z|>c_{1}}|z|^{q} g_{\mathrm{L}}(z) d z \\
& =t^{q / \alpha} \int_{-\infty}^{\infty}|y|^{q} g_{\mathrm{B}}(y) d y & & +O\left[t^{-(\alpha-1)+q}\right] \\
& \sim M_{q}^{<} t^{q / \alpha} \quad(0<q<\alpha) &
\end{array}
$$

When going from the first to the second line, we used the fact that $g_{\mathrm{B}}(y)$ is $\alpha$-stable, and therefore the related integral converges with time to the finite moment $M_{q}^{<}$of order $q<\alpha$. In the third line, the bulk contribution dominates at large times, since we have the relations

$$
-(\alpha-1)+q \lessgtr q / \alpha \stackrel{\alpha>1}{\Longleftrightarrow} q \lessgtr \alpha .
$$

\section{High-order moments, $q>\alpha$.}

We choose $c(t) \equiv t^{1 / \alpha} c_{2}$, with some $\kappa \ll c_{2}$, and continue Eq. (B-B2):

$$
\begin{aligned}
\left\langle\left|\Delta T_{x}(t)\right|^{q}\right\rangle & \sim t^{q / \alpha} \int_{|y| \leq c_{2}}|y|^{q} g_{\mathrm{B}}(y) d y & +t^{-(\alpha-1)+q} \int_{|z|>t^{1 / \alpha-1} c_{2}}|z|^{q} g_{\mathrm{L}}(z) d z \\
& =O\left[t^{q / \alpha}\right] & +t^{-(\alpha-1)+q} \int_{-P_{x}}^{1-P_{x}}|z|^{q} g_{\mathrm{L}}(z ; 1) d z \\
& \sim M_{q}^{>} t^{-(\alpha-1)+q} \quad(q>\alpha) &
\end{aligned}
$$

The integral on the second line gives the value of $M_{q}^{>}$as in Eq. (27). We stress that the latter is finite: the infinite density $g_{\mathrm{L}}(z)$ diverges at the origin like $|z|^{-\alpha-1}$, but the factor $|z|^{q}$ guarantees convergence for any $q>\alpha$. Finally, due to relation (B4), the large fluctuations with time dependence $t^{-(\alpha-1)+q}$ dominate higher order moments $q>\alpha$.

\section{Borderline case, $q=\alpha$}

We set again $c(t) \equiv t^{1 / \alpha} c_{2}, c_{2} \gg \kappa$. For $q=\alpha$, both bulk and large fluctuations contribute to moments in the same way. This can be seen as follows.

$$
\begin{aligned}
\left\langle\left|\Delta T_{x}(t)\right|^{\alpha}\right\rangle & \sim t \int_{|y| \leq c_{2}}|y|^{\alpha} g_{\mathrm{B}}(y) d y+t \int_{|z|>t^{1 / \alpha-1} c_{2}}|z|^{\alpha} g_{\mathrm{L}}(z) d z \\
& =O[t]-t \frac{C \alpha}{|\Gamma(1-\alpha)|}\left[\left(1-P_{x}\right)^{\alpha} P_{x} \ln \left(t^{1 / \alpha-1} c_{2}\right)+\left(1-P_{x}\right) P_{x}^{\alpha} \ln \left(t^{1 / \alpha-1} c_{2}\right)\right] \\
& \sim \frac{C(\alpha-1)}{|\Gamma(1-\alpha)|}\left[\left(1-P_{x}\right)^{\alpha} P_{x}+\left(1-P_{x}\right) P_{x}^{\alpha}\right] t \ln t
\end{aligned}
$$

Since $|z|^{\alpha} g_{\mathrm{L}}(z ; 1)$ behaves at the origin as $|z|^{-1}$, the large fluctuations integral on the first line diverges logarithmically as $t \rightarrow \infty$. This is why in this case, we had to consider explicitly the form of the power law pole, Eq. (26). Terms of the order of $t$, were neglected on the last line. This includes in particular the contributions from the very center of the stable density $\left(|y| \leq c_{2}\right)$ and the extremities of the infinite density $\left(z \approx-P_{x}\right.$ and $\left.z \approx 1-P_{x}\right)$ and the $c_{2}$-dependence of function $c(t)$. Hence, in a sense, only the power law overlap between the Lévy-stable and the infinite density determines the asymptotic form of the moment $q=\alpha$. 


\section{Appendix C: Fluctuations statistics of general time averages, Eqs. (32)-36}

We outline here the derivation of the long-time asymptotics of the PDF $h(\overline{\Delta o} ; t)$ for the fluctuations $\overline{\Delta \mathcal{O}}(t)$ of the time average of a general observable $\mathcal{O}$. Our starting point is the joint $\operatorname{PDF} f(\bar{o}, n ; t)$ for measuring the $\overline{\mathcal{O}}(t)=\bar{o}$ at time $t$, while having made exactly $N(t)=n$ random walk steps up to that point. We can write down its Fourier-Laplace representation as

$$
\widehat{f}(u, n ; s)=\int_{0}^{\infty} e^{-s t}\langle\exp [-u t \overline{\mathcal{O}}(t)] \delta[N(t)=n]\rangle d t
$$

The average $\langle\cdot\rangle$ is to be interpreted here as an average over all realizations of the CTRW process. According to Ref. [39], Sec. 5.3, one can write

$$
\begin{aligned}
& \widehat{f}(u, n ; s)= \\
& =\left\langle\frac{1-\widehat{\psi}\left(s+u o_{X}^{\mathrm{RW}}(n)\right)}{s+u o_{X} \mathrm{RW}_{(n)}} \prod_{x}\left[\widehat{\psi}\left(s+u o_{x}\right)\right]^{N_{x}^{\mathrm{RW}}(n)}\right\rangle .
\end{aligned}
$$

This should be read as follows. The average with respect to the random, independent and identically distributed waiting times has already been carried out. The statistics of waiting times is embedded via the Laplace transform $\widehat{\psi}(s)$. The remaining average is to be taken with respect to two aspects of the trajectories of an analogue ordinary random walk (RW), i.e. where the time between steps is fixed to unity. First, we have to average over all terminal positions $X^{\mathrm{RW}}(n)$ after $n$ steps. Second, we average over the possible sets of visitation numbers $N_{x}^{\mathrm{RW}}(n)$, i.e. the number of times an ordinary random walker has visits each of the sites $x$ of the lattice within $n$ steps.

We want to approximate Eq. (C2) in the limit of large times and, consequently, large values of $t \overline{\mathcal{O}}(t)$. This translates to small $s, u$. On the one hand, for the broadtailed waiting times as in Eq. (3), this means we expand

$$
\widehat{\psi}(s+u \mathcal{O}) \sim 1-\langle\tau\rangle(s+u \mathcal{O})+A(s+u \mathcal{O})^{\alpha}
$$

On the other hand, it is natural to assume that we need to concentrate on large $n$, so we require the statistics of the ordinary random walk after many steps. Since we confine our discussion to ergodic random walks, it is easy to say what happens when $n$ becomes infinite. The position $X^{\mathrm{RW}}(n)$ is distributed according to the $n$ independent equilibrium distribution $P_{x}$, while the visitation numbers converge as $N^{\mathrm{RW}}(n) / n \rightarrow P_{x}$. At finite $n$, deviations from these ergodic limits appear. However, for ordinary random walks, these kind of fluctuations do typically not exhibit broad-tailed statistics. We rather assume that their contribution in Eq. (C2) is of the or$\operatorname{der} O\left[(s+u \mathcal{O})^{2}\right]$. They are then negligible as compared to the fluctuations caused by the broad-tailed waiting times $\mathrm{C3}$. Recall that in the specific case of the site occupation times $\Delta T_{x}(t)$, Sec. IV, we gave a more rigorous argument for this assumption in terms of a first-passage time analysis. Only the mean of the first-passage time of the ordinary random walk plays a role in the asymptotics of occupation time fluctuations. For this or similar arguments to hold, it is obviously crucial that $\alpha<2$.

We thus now approximate Eq. (C2) by using Eq. (C3), by writing the average with respect to $X^{\mathrm{RW}}(n)$ as an equilibrium average and by replacing the visitation numbers $N^{\mathrm{RW}}(n)$ by their ergodic limit values $n P_{x}$. This yields

$$
\begin{aligned}
\widehat{f}(u, n ; s) \sim & \\
\sim & \left\langle\frac{\langle\tau\rangle(s+u \mathcal{O})-A(s+u \mathcal{O})^{\alpha}}{s+u \mathcal{O}}\right\rangle_{\mathrm{eq}} \times \\
& \prod_{x}\left[1-\langle\tau\rangle\left(s+u o_{x}\right)+A\left(s+u o_{x}\right)^{\alpha}\right]^{n P_{x}} \\
= & {\left[\langle\tau\rangle-A\left\langle(s+u \mathcal{O})^{\alpha-1}\right\rangle_{\mathrm{eq}}\right] \times } \\
& \exp \left\{n \sum_{x} P_{x} \ln \left[1-\langle\tau\rangle\left(s+u o_{x}\right)+A\left(s+u o_{x}\right)^{\alpha}\right]\right\} .
\end{aligned}
$$

At this point, we marginalize out the number of steps. Since we are operating in a limit where $n$ is assumed large, we find it appropriate to treat $n$ as continuous and integrate, that is

$$
\begin{aligned}
\widehat{f}(u ; s) & \sim \int_{0}^{\infty} \widehat{f}(u, n ; s) d n= \\
& =\frac{\langle\tau\rangle-A\left\langle(s+u \mathcal{O})^{\alpha-1}\right\rangle_{\mathrm{eq}}}{\sum_{x} P_{x} \ln \left[1-\langle\tau\rangle\left(s+u o_{x}\right)+A\left(s+u o_{x}\right)^{\alpha}\right]} \\
& \sim \frac{1-C\left\langle(s+u \mathcal{O})^{\alpha-1}\right\rangle_{\mathrm{eq}}}{s+u\langle\mathcal{O}\rangle_{\mathrm{eq}}-C\left\langle(s+u \mathcal{O})^{\alpha}\right\rangle_{\mathrm{eq}}}
\end{aligned}
$$

In the last step, we used $\ln (1+x) \sim x+O\left[x^{2}\right]$ for small $x$. Equation (C5) is the generalization of Eq. (12). The latter can be recovered by considering the occupation observable in Eq. (31).

The PDF of the fluctuations $\overline{\Delta \mathcal{O}}(t)$ are most suitably discussed in Fourier-Laplace space. By shifting by $-t\langle\mathcal{O}\rangle_{\text {eq }}$, we get

$$
\begin{aligned}
\widehat{h}(k ; t) & =\int_{0}^{\infty}\langle\exp [i k t \overline{\Delta \mathcal{O}}(t)]\rangle d t=\widehat{f}\left(-i k ; s+i k\langle\mathcal{O}\rangle_{\mathrm{eq}}\right) \\
& \sim \frac{1-C\left\langle(s-i k \Delta \mathcal{O})^{\alpha-1}\right\rangle_{\mathrm{eq}}}{s-C\left\langle(s-i k \Delta \mathcal{O})^{\alpha}\right\rangle_{\mathrm{eq}}},
\end{aligned}
$$

generalizing Eq. (13). By methods which are completely analogous to those used in Sec. $\mathrm{V}]$ one can compute the limit where $s$ becomes small while $s /|k|^{\alpha}$ is fixed. This gives the Lévy $\alpha$-stable bulk statistics of the fluctuations of time averages, Eqs. (34)-35). The limit where $s$ is small and $s /|k|$ fixed is obtained through the procedure 
discussed in Sec. VI and A It yields the infinite density of large fluctuations, Eq. (36).
[1] N. Agmon, Chem. Phys. Lett. 497, 184 (2010).

[2] A. M. Berezhkovskii, V. Zaloj, and N. Agmon, Phys. Rev. E 57, 3937 (1998).

[3] O. Bénichou, M. Coppey, J. Klafter, M. Moreau, and G. Oshanin, J. Phys. A 367225 (2003).

[4] C. Godrèche and J. M. Luck, J. Stat. Phys. 104, 489 (2001).

[5] S. N. Majumdar, Curr. Sci. 77, 370 (1999).

[6] F. Bardou, J.-P. Bouchaud, A. Aspect, and C. CohenTannoudji, Lévy Statistics and Laser Cooling (Cambridge University Press, Cambridge, UK, 2002).

[7] F. Bardou, J.-P. Bouchaud, O. Emile, A. Aspect, and C. Cohen-Tannoudji, Phys. Rev. Lett. 72, 203 (1994).

[8] C. Jarzynski, Annu. Rev. Condens. Matter Phys. 2, 329 (2011) Thermodynamics at the Nanoscale

[9] U. Seifert, Rep. Prog. Phys. 75, 126001 (2012).

[10] C. Monthus, and J.-P. Bouchaud, J. Phys. A 29, 3847 (1996)

[11] G. Bel, and E. Barkai, Phys. Rev. Lett. 94, 240602 (2005).

[12] G. Bel, and E. Barkai, Phys. Rev. E 73, 016125 (2006)

[13] S. N. Majumdar, and A. Comtet, Phys. Rev. Lett. 89, 060601 (2002).

[14] S. Burov, and E. Barkai, Phys. Rev. Lett. 98, 250601 (2007)

[15] H. Scher and E. W. Montroll, Phys. Rev. B 12, 2455 (1975).

[16] E. Lutz, Phys. Rev. Lett. 93, 190602 (2004).

[17] J.-H. Jeon, V. Tejedor, S. Burov, E. Barkai, C. SelhuberUnkel, K. Berg-Sørensen, L. Oddershede, and R. Metzler, Phys. Rev. Lett. 106, 048103 (2011).

[18] A. V. Weigel, B. Simon, M. M. Tamkun, and D. Krapf, PNAS 108, 6438 (2011). single-molecule tracking

[19] Q. Xu, L. Feng, R. Sha, N. C. Seeman, and P. M. Chaikin, Phys. Rev. Lett. 106, 228102 (2011).

[20] I. Y. Wong, M. L. Gardel, D. R. Reichman, E. R. Weeks, M. T. Valentine, A. R. Bausch, and D. A. Weitz, Phys. Rev. Lett. 92, 178101 (2004). Networks

[21] J.-P. Bouchaud, A. Georges, Phys. Rep. 195, 127 (1990) PHYSICAL APPLICATIONS

[22] R. Metzler and J. Klafter, Phys. Rep. 339, 1 (2000).

[23] R. Metzler and J. Klafter, J. Phys. A 37, R161 (2004). description of anomalous transport by fractional dynamics

[24] R. Metzler, J.-H. Jeon, A. G. Cherstvy, and E. Barkai, Phys. Chem. Chem. Phys. 16, 24128 (2014) nonergodicity, and ageing at the centenary of single particle tracking

[25] B. D. Hughes, Random Walks and Random Environments, Vol. I: Random Walks (Oxford University Press,
Oxford, UK, 1995).

[26] E. Bertin, and F. Bardou, Am. J. Phys. 76, 630 (2008).

[27] M. Thaler, Israel J. Math. 46, 67 (1983). points

[28] M. Thaler and R. Zweimüller, Probability theory and related fields 135, 15 (2006).

[29] J. Aaronson, An Introduction to Infinite Ergodic Theory (American Mathematical Society, Providence, 1997).

[30] N. Korabel, and E. Barkai, Phys. Rev. Lett. 102, 050601 (2009).

[31] N. Korabel, and E. Barkai, Phys. Rev. Lett. 108, 060604 (2012). Chaos

[32] T. Akimoto, and T. Miyaguchi, Phys. Rev. E. 82, 030102(R) (2010).

[33] T. Akimoto, Phys. Rev. Lett. 108, 164101 (2012).

[34] D. A. Kessler, and E. Barkai, Phys. Rev. Lett. 105, 120602 (2010). Lattices

[35] P. C. Holz, A. Dechant, and E. Lutz, Europhys. Lett. 109, 23001 (2015).

[36] E. Lutz and F. Renzoni, Nature Phys. 9, 615 (2013).

[37] A. Rebenshtok, S. Denisov, P. Hänggi, and E. Barkai, Phys. Rev. Lett. 112, 110601 (2014), Limit Theorem ibid. Phys. Rev. E 90, 062135 (2014).

[38] J. H. P. Schulz, E. Barkai, and R. Metzler, Phys. Rev. X 4, 011028 (2014).

[39] A. Rebenshtok, and E. Barkai, J. Stat. Phys. 133, 565 (2008).

[40] S. Burov, R. Metzler, and E. Barkai, PNAS 107, 13228 (2010).

[41] M. Kac, Probability and Related Topics in Physical Sciences (Interscience, New York, 1958).

[42] G. M. Zaslavsky, Phys. Rep. 371, 461 (2002).

[43] B. V. Gnedenko and A. N. Kolmogorov, Limit Distributions for Sums of Independent Random Variables (Addison-Wesley, Cambridge, MA, 1954).

[44] We note that Eq. (8) is derived under the assumption that at time $t=0$, the random walker is not residing on $x$. However, in the large time limit, the choice of this initial condition has no effect (see Ref. [12]).

[45] O. Bénichou, and R. Voituriez, Physics Reports 539, 225 (2014). kinetics

[46] The authors of Ref. [45] use a definition of the first-return time $\bar{\tau}_{\mathrm{n} x}^{\mathrm{RW}}$ which slightly differs from ours: it includes the time spent at the site $x$ before jumping off. Hence, for us, $\tau_{\mathrm{n} x}^{\mathrm{RW}}=\bar{\tau}_{\mathrm{n} x}^{\mathrm{RW}}-1$.

[47] G. Samorodnitsky and M. S. Taqqu, Stable NonGaussian Random Processes: Stochastic Models with Infinite Variance (Chapman \& Hall, 1994).

[48] P. Castiglione, A. Mazzino, P. Muratore-Ginanneschi, and A. Vulpiani, Physica D (Amsterdam) 134, 75 (1999).

[49] H. Touchette, Phys. Rep. 478, 1 (2009). 